\title{
Farrerol ameliorates diabetic hepatopathy in rat model of type 2 diabetes mellitus via modulation of oxidative- inflammatory stress
}

\author{
Li Dong ${ }^{1}$, Lixia Yang ${ }^{2}$, Fengsui Liu ${ }^{1 \star}$, Haitao Zhan ${ }^{1}$, Xinwei Chen ${ }^{1}$ \\ ${ }^{1}$ Department of Critical Care Medicine, ${ }^{2}$ Department of Quality Management, First Hospital of Qinhuangdao, Qinhuangdao \\ 066001, Hebei, China
}

*For correspondence: Email: LucySantanawua@yahoo.com; Tel: 0086-0335-3032042

\begin{abstract}
Purpose: To investigate the effect of farrerol on diabetic hepatopathy in a rat model of type 2 diabetes mellitus (T2DM).

Methods: Adult male Wistar rats $(n=40)$ were randomly assigned to four groups of ten rats each: normal control, diabetic control, farrerol control and treatment groups. With the exception of normal control and farrerol control groups, the rats were fed high-fat diet (HFD) for four weeks, and thereafter injected streptozotocin (STZ) at a dose of $30 \mathrm{mg} / \mathrm{kg}$ body weight intraperitoneally (i.p.) for induction of T2DM. Rats in farrerol control and treatment groups received $50 \mathrm{mg} / \mathrm{kg}$ farrerol orally/day. Serum levels of triacylglycerol (TG), total cholesterol (TC), high-density lipoprotein cholesterol (HDL-C) and lowdensity lipoprotein cholesterol (LDL-C) were determined. Superoxide dismutase (SOD) activity and malondialdehyde (MDA) levels were assessed in liver homogenate while MRNA and protein expressions of glucose transporter 2 (GLUT2) were assayed in liver using real-time quantitative polymerase chain reaction ( $q R T-P C R$ ) and Western blotting, respectively. Expression levels of tumor necrosis factor- $\alpha$ (TNF- $\alpha$ ) and interleukin-1 $\beta$ (IL-1 $\beta$ ) were also determined using qRT-PCR.

Results: Diabetes mellitus (DM) led to significant reductions in rat body weight and SOD activity, while increasing fasting blood glucose $(F B G)$ and MDA levels $(p<0.05)$. However, treatment with farrerol significantly reversed the effect of DM on these parameters $(p<0.05)$. The mRNA expressions of TNF$\alpha$ and IL-1 $\beta$ were significantly higher in diabetic control group than in normal control group, but were significantly reduced after farrerol treatment $(p<0.05)$. Treatment with farrerol also significantly reversed the effect of DM on rat lipid profile $(p<0.05)$. The expression of GLUT2 protein was significantly downregulated in the liver of diabetic control rats, when compared with normal control rats, but was significantly upregulated after treatment with farrerol $(p<0.05)$.

Conclusion: The results of this study show that farrerol alleviates STZ-induced hyperglycemia and dyslipidemia via reduction in oxidative stress and inflammation, and upregulation of GLUT2 protein expression. Thus, farrerol has antidiabetic and hepatoprotective potentials for clinical use in humans.
\end{abstract}

Keywords: Diabetes mellitus, Dyslipidemia, Farrerol, Hepatopathy, High-fat diet

\begin{abstract}
This is an Open Access article that uses a fund-ing model which does not charge readers or their institutions for access and distributed under the terms of the Creative Commons Attribution License (http://creativecommons.org/licenses/by/4.0) and the Budapest Open Access Initiative (http://www.budapestopenaccessinitiative.org/read), which permit unrestricted use, distribution, and reproduction in any medium, provided the original work is properly credited.
\end{abstract}

Tropical Journal of Pharmaceutical Research is indexed by Science Citation Index (SciSearch), Scopus, International Pharmaceutical Abstract, Chemical Abstracts, Embase, Index Copernicus, EBSCO, African Index Medicus, JournalSeek, Journal Citation Reports/Science Edition, Directory of Open Access Journals (DOAJ), African Journal Online, Bioline International, Open-J-Gate and Pharmacy Abstracts 


\section{INTRODUCTION}

Diabetes mellitus (DM) is a heterogeneous group of syndromes characterized by elevation of FBG caused by a relative or absolute deficiency of insulin. In 2018, the global prevalence of T2DM was about half a billion, thereby creating financial and psychological hardship for sufferers [1]. There is a direct relationship between DM and liver disease, and the combined prevalence of co-morbidity is higher than the that associated with a single condition. Hepatic complications of DM are not easily noticeable. Pre-clinical and epidemiological evidence indicate a clinical link between DM and liver diseases such as nonalcoholic steatohepatitis (NASH), non-alcoholic fatty liver disease (NAFLD), liver cirrhosis and metastatic hepatocellular carcinoma [2]. Nonalcoholic fatty liver is a common co-morbidity in approximately $70 \%$ of patients with T2DM [3]. This explains the persistent occurrence of obesity and insulin resistance in diabetics.

At present, therapeutic agents such as dipeptidyl dipeptidase-4 inhibitor (DDP-4i) and glucagonlike peptide-1 (GLP-1) mimics, and peroxisome proliferator-activated receptor gamma (PPAR- $\mathrm{Y}$ ) agonists have shown potential as effective antidiabetic agents [4]. For instance, the combination of pioglitazone (PPAR-y modulator) with vitamin $E$ has been shown to alleviate diabetic complications [4]. In addition, combination of pioglitazone with statins confers8 protection on cardiovascular function. However, the problem of adverse effect remains a major challenge. Therefore, the search for safe and effective therapies with little or no adverse effects has become necessary.

Plant-derived compounds are important sources of drugs. They are relatively safe and effective. Farrerol, a bio-flavanone isolated from Rhododendron dauricum L. exhibits antioxidant, anti-inflammatory, hepatoprotective and cardioprotective effects $[5,6]$. At present, little or nothing is known about its antidiabetic and hepatoprotective effects in patients with diabetic hepatopathy. This study investigated the effect of farrerol on rat model of T2DM.

\section{EXPERIMENTAL}

\section{Materials}

Farrerol, STZ and polyvinylidene difluoride (PVDF) membrane were purchased from SigmaAldrich (USA). High-fat diet-containing sucrose and lard (10\%), $1 \%$ cholesterol, and $0.3 \%$ sodium cholate were obtained from HFK Technology Co. Ltd (China). Blood glucose test strips and glucometer were products of Beijing Yicheng Bioelectronics Technology Co. Ltd (China); lipid profile kits were obtained from NanJing JianCheng Bioengineering Institute (China). Automated biochemical analyzer was a product of Hitachi Ltd. (Japan). Superoxide dismutase (SOD) and MDA assay kits were purchased from Bio-diagnostic (Egypt); bicinchoninic acid (BCA) protein kit was obtained from Bio-Rad Laboratories (USA), while GLUT2 primary antibody was purchased from Cayman Chemicals (USA). Horseradish peroxidase (HRP)-conjugated secondary antibodies were products of Santa Cruz Biotechnology, Inc (USA). Total RNA isolation reagent was purchased from BIOER (China).

\section{Experimental rats and treatment regimen}

Adult male Wistar rats $(n=40)$ weighing 190 $220 \mathrm{~g}$ (mean weight $=205 \pm 15 \mathrm{~g}$ ) were used in this study. The rats were exposed to $12 \mathrm{~h}$ light/12 $\mathrm{h}$ dark cycles and maintained at a temperature of $25{ }^{\circ} \mathrm{C}$, and $50 \%$ humidity in an aseptic environment. They were acclimatized for seven days before commencement of the study, and had free access to standard feed and clean drinking water. The rats were randomly assigned to four groups of ten rats each: normal control group, diabetic control group, farrerol control group and treatment group. With the exception of normal control and farrerol control groups, the rats were fed HFD for four weeks, and T2DM was induced through injection with STZ at a dose of $30 \mathrm{mg} / \mathrm{kg} \mathrm{STZ} \mathrm{i.p.} \mathrm{The} \mathrm{STZ} \mathrm{was} \mathrm{prepared} \mathrm{in}$ citrate buffer. Rats in the farrerol control and treatment groups received ferrarol at a dose of $50 \mathrm{mg} / \mathrm{kg}$ orally/day. Fasting blood glucose (FBG) and body weight of the rats were measured at the beginning and end of the study. The study protocol was approved by the Institutional Animal Ethics Committee of the First Hospital of Qinhuangdao (approval no. 20170338), and it adhered strictly to the National Institute of Health (NIH) guidelines for the care and use of laboratory animals [7].

\section{Biochemical assays}

At the end of the treatment period, the rats were euthanized and blood was collected in plain sample containers. The blood was centrifuged at $3000 \mathrm{rpm}$ for $10 \mathrm{~min}$ to obtain serum which was used for biochemical analyses. The liver was also excised and used to prepare $20 \%$ tissue homogenate. The biochemical parameters measured were TC, TG, HDL-C and LDL-C. The excised liver was homogenized using a Teflon homogenizer and the resultant homogenate was centrifuged at $3000 \mathrm{rpm}$ for $10 \mathrm{~min}$ to obtain 
supernatant which was used for determination of SOD activity and MDA levels.

\section{qRT-PCR}

Trizol RNA extraction reagent was used to extract total RNA from cell suspension resulting from the trysinization of the liver, while complementary DNA (cDNA) synthesis kit was used to perform reverse transcription for cDNA synthesis according to the instructions of the manufacturer. Light Cycler 1536 T-PCR detection system was used for the estimation of the mRNA expressions of $\mathrm{IL}-1 \beta$ and TNF- $\alpha$. Variation in the cDNA content was normalized using $\beta$-actin. The PCR reaction mixture $(20 \mu \mathrm{L})$ consisted of $6.4 \mu \mathrm{L}$ of $\mathrm{dH}_{2} \mathrm{O}, 1.6 \mu \mathrm{L}$ of genespecific primer $(10 \mu \mathrm{M}), 2 \mu \mathrm{L}$ of synthesized cDNA and $10 \mu \mathrm{L}$ of SYBR Premix Ex Taq ${ }^{\mathrm{TM}}$ II. The Ct value of $\mathrm{U} 6$ was taken as the internal parameter, and $2^{-\Delta \Delta \mathrm{Ct}}$ was used to calculate the relative expression levels of the proteins. The primer sequences used for qRT-PCR are shown in Table 1.

Table 1: Primer sequences used for qRT-PCR

\begin{tabular}{ll}
\hline Gene & Sequence \\
\hline$\beta$-actin & Forward: \\
& ATGACGATATCGCTGCGCTC \\
& Reverse: \\
& TACCCACCACACACCCTGG \\
TNF- $\alpha$ & Forward: \\
& TCCCAGGTTCTCTTCAAGG \\
& Reverse: \\
& GTACATGGGCTCATACCAG \\
$\mathrm{IL}-1 \beta$ & Forward: \\
& GGACAGAACATAAGCCAACA \\
& Reverse: \\
& CTTTCATCACACAGGACAGG \\
\hline
\end{tabular}

\section{Western blotting}

The liver was trypsinized using $0.25 \%$ trypsin, and the resultant cell suspension was washed with phosphate-buffered saline (PBS). Ice-cold radio-immunoprecipitation assay (RIPA) buffer containing protease inhibitor was used to lyse the cells. The resultant lysate was centrifuged at 16 , $000 \mathrm{rpm}$ for $20 \mathrm{~min}$ at $4{ }^{\circ} \mathrm{C}$, and the protein concentration of the supernatant was determined using BCA assay kit. A portion of total cell protein $(50 \mu \mathrm{g})$ from each sample was separated on 12 $\%$ sodium dodecyl sulphate (SDS)polyacrylamide gel electrophoresis and transferred to a fixed polyvinylidene fluoride membrane at $110 \mathrm{~V}$ and $90{ }^{\circ} \mathrm{C}$ for $120 \mathrm{~min}$. Subsequently, non-fat milk powder $(3 \%)$ in Trisbuffered saline containing $0.2 \%$ Tween-20 (TBST) was added with gentle shaking at $37{ }^{\circ} \mathrm{C}$ and incubated to block non-specific binding of the blot. Incubation of the blots was performed overnight at $4{ }^{\circ} \mathrm{C}$ with primary antibodies of GLUT2 and $\beta$-actin, each at a dilution of 1:1000. Then, the membrane was washed thrice with TBS-T, and further incubated with horseradish peroxidase-conjugated goat anti-rabbit IgG secondary antibody for $1.5 \mathrm{~h}$ at room temperature. The blot was developed using an Xray film. Grayscale analysis of the bands was performed using Tanon imaging system. The respective protein expression levels were normalized to that of $\beta$-actin which was used as a standard.

\section{Statistical analysis}

Data are expressed as mean \pm SD. Statistical analysis was performed using SPSS (13.0). Groups were compared using Student's $t$-test. Statistical significance was assumed at $p<0.05$.

\section{RESULTS}

\section{Effect of farrerol on body weight and FBG levels of rats}

Treatment of diabetic rats with farrerol significantly increased the body weights of the rats, but significantly reduced their FBG levels ( $p$ $<0.05)$. These results are shown in Figures 1 and 2 .

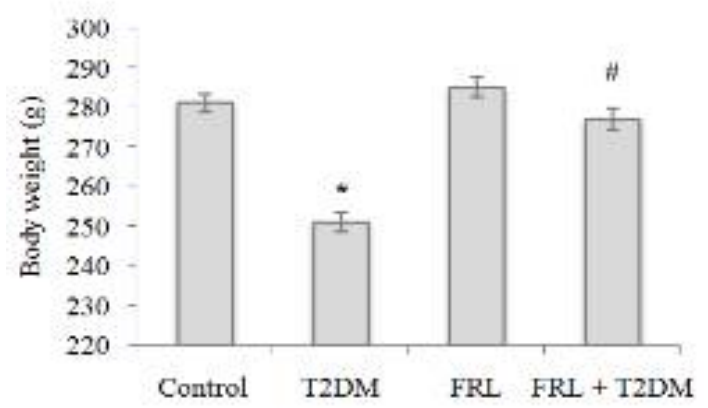

Figure 1: Changes in body weights of rats after farrerol treatment; $p<0.05$, when compared with normal control group; and ${ }^{\#} p<0.05$, when compared with diabetic control group

\section{Effects of farrerol on oxidative status in diabetic rats}

As shown in Figure 3 and Figure 4, treatment of diabetic rats with farrerol significantly increased SOD activity, while reducing the level of MDA ( $p$ $<0.05)$.

\section{Effect of farrerol on the expression levels of pro-inflammatory markers in diabetic rats}

As shown in Figure 5, the mRNA expressions of TNF- $\alpha$ and IL-1 $\beta$ were significantly higher in 
diabetic control group than in normal control group $(p<0.05)$. However, treatment with farrerol significantly reversed the effect of DM on the mRNA expressions of these pro-inflammatory cytokines $(p<0.05)$.

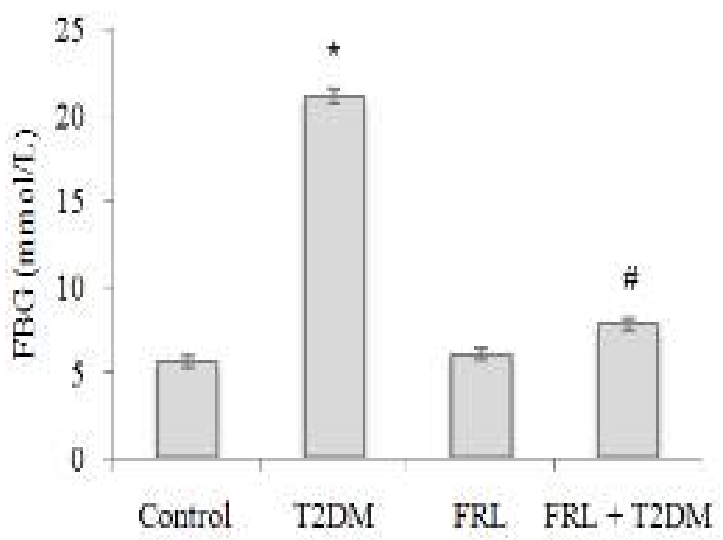

Figure 2: Effect of farrerol on FBG levels; ${ }^{*} p<0.05$, when compared with normal control group; and ${ }^{\#} p<$ 0.05 when compared with diabetic control group

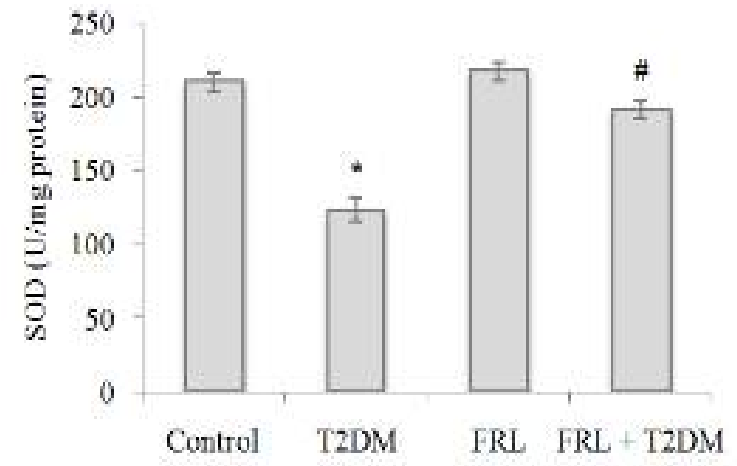

Figure 3: Activity of SOD after treatment of diabetic rats with farrerol; " $p<0.05$, when compared with normal control group; and ${ }^{\#} p<0.05$ when compared with diabetic control group

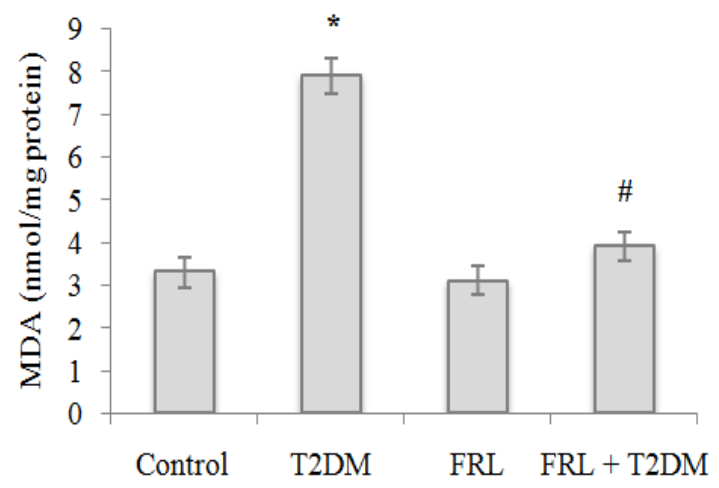

Figure 4: Effect of farrerol on MDA level of diabetic rats; " $p<0.05$, when compared with normal control group; and ${ }^{\#} p<0.05$ when compared with diabetic control group

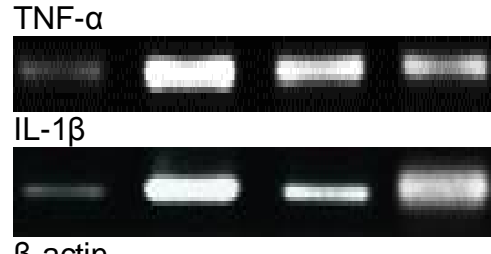

$\beta$-actin
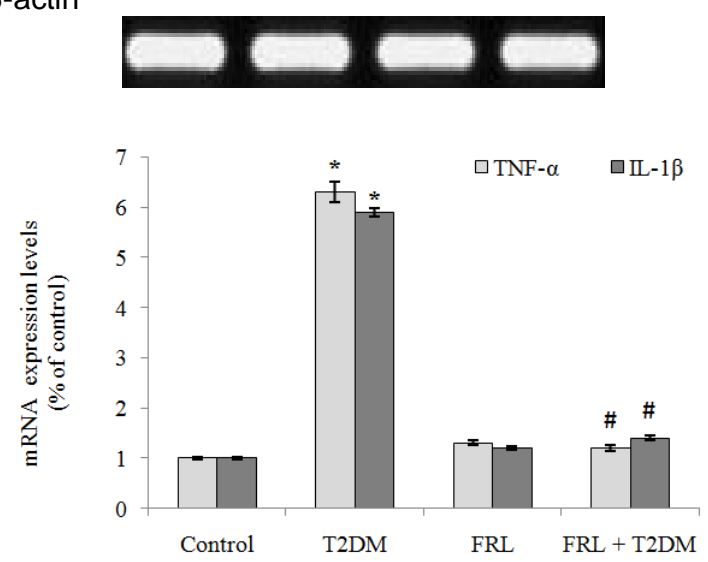

Figure 5: Effect of farrerol on levels of expression of inflammatory cytokines in the liver of diabetic rats; $p<$ 0.05 , when compared with normal control group; and $\# p<0.05$ when compared with diabetic control group

\section{Effect of farrerol on lipid profile of diabetic rats}

Treatment of diabetic rats with farrerol significantly improved their lipid profile $(p<0.05$; Figure 6).

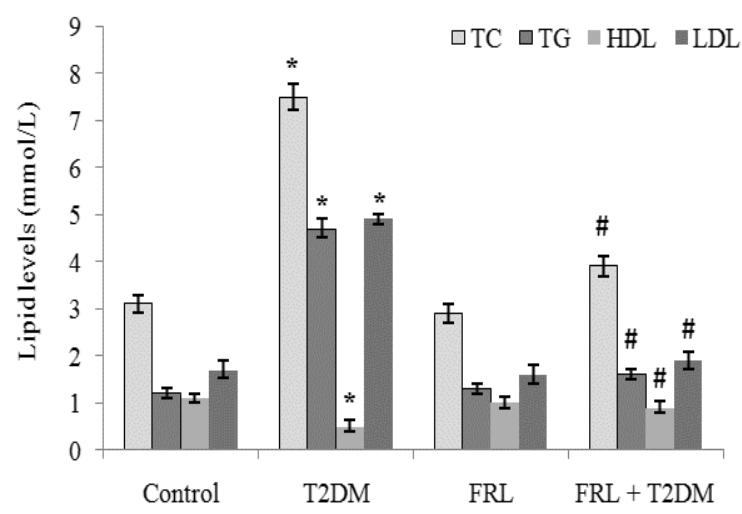

Figure 6: Effect of farrerol on lipid profile of diabetic rats; ${ }^{*} p<0.05$, when compared with normal control group; ${ }^{*} p<0.05$ when compared with diabetic control group

\section{Effect of farrerol on GLUT2 protein expression in diabetic rats}

The expression of GLUT2 protein was significantly down-regulated in the liver of diabetic control rats, when compared with normal control rats, but was significantly upregulated after treatment with farrerol $(p<0.05$; Figure 7$)$. 
GLUT2

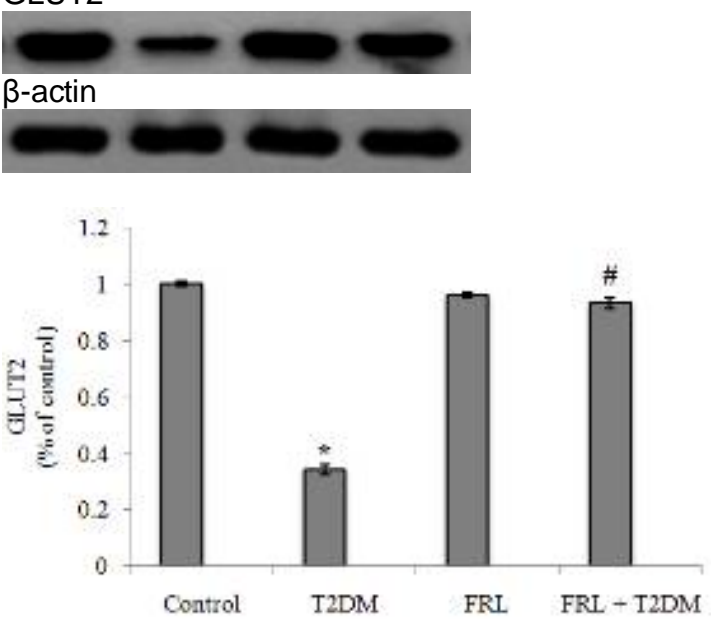

Figure 7: Effect of farrerol on the expression of GLUT2 in diabetic rats; ${ }^{*} p<0.05$, when compared with normal control group; and ${ }^{\#} p<0.05$ when compared with diabetic control group

\section{DISCUSSION}

Obesity is a major risk factor for T2DM. The combination of HFD with intraperitoneal injection of low-dose STZ is a common pre-clinical model for studying T2DM. In this model, HFD triggers insulin resistance, while STZ reduces pancreatic beta-cell population, thereby producing persistent hyperglycemia. In this study, HFD-low-dose STZ administration significantly elevated FBG level, and resulted in substantial weight loss. However, farrerol treatment significantly reversed these metabolic derangements. Studies have shown that farrerol suppresses the activity of nicotinamide adenine dinucleotide phosphate (NADPH) oxidase, a pro-oxidant enzyme and key regulator of redox homeostasis in insulinsensitive tissues $[5,8]$.

Oxidative stress in T2DM is an important pathological state caused by hyperglycemia and generation of reactive oxygen species (ROS). An upsurge in ROS elicits a chain reaction between free-radicals and membrane-bound free-fatty acids (FFAs) in an insulin-sensitive tissue like liver. This in turn, results in the formation of noxious lipid peroxidation products such as hydroxynonenal (HNE) and MDA, which provoke the release of pro-inflammatory cytokines [9]. An increase in hepatic lipid peroxidation is indicative of reduced inherent antioxidant defense system in the liver. Oxidative stress plays an important role in apoptosis and tissue degeneration.

Superoxide dismutase (SOD) is the principal antioxidant enzyme in cells. In this study, SOD activity was significantly reduced, while MDA level was significantly elevated in hepatic tissue of diabetic rats. However, farrerol treatment significantly ameliorated these alterations.

These results are in agreement with those of previous reports. It is likely that the observed effect of farrerol was exerted via Nrf2-ARE activation [6]. Studies have shown that activation of the Nrf2-ARE signaling pathway prevents hyperglycemia-induced oxidative mitochondrial injury [10].

Diabesity is a modern epidemic which indicates the coexistence of both diabetes and obesity. It is linked to various pathophysiological mechanisms revolving around insulin resistance and hyperinsulinemia. According to the "Inflammation Hypothesis", obesity is a corollary of the unremitting inflammatory process caused by macrophage infiltration into adipose tissue and the resultant detrimental metabolic derangements in insulin-sensitive tissues and pancreatic beta cells [11]. The combination of HFD and necro-inflammation is the pathological initiator of insulin resistance and T2DM. Studies have shown that HFD promotes mitochondrial ROS accretion, which triggers NLRP3-ASC inflammasome, and ultimately insulin resistance [12]. It has also been reported that increased synthesis of inflammatory cytokines such as IL$1 \beta$ and TNF- $\alpha$ in diabetic hepatopathy is mediated through TLR4/MyD88/NF- $\kappa$ B pathway [13]. An elevation in IL-1 $\beta$ level promotes the synthesis of TNF- $\alpha$ and exacerbation of insulin resistance. In this study, IL-1 $1 \beta$ and TNF- $\alpha$ mRNA levels were significantly elevated in diabetic rats, but were significantly reduced after treatment with farrerol.

Excessive consumption of HFD causes oxidative outburst which promotes protein kinase $\mathrm{C}$ (PKC)mediated impairment of hepatocyte nuclear factor $4 \alpha(\mathrm{HNF} 4 \alpha)$, a key regulator of hepatic triacylglycerol and cholesterol homeostasis [13]. In this study, serum TG, TC and LDL-C levels were significantly increased in the diabetic rats, while HDL-C level was significantly reduced. However, farrerol treatment significantly reversed the effect of DM on rat lipid profile.

Glucose transporter 2 (GLUT2) is the principal glucose transporter. Genetic /epigenetic alterations in protein expression of GLUT2 transporter affect glucose-induced insulin secretion and uptake by hepatocytes. In this study, the expression of GLUT2 protein was significantly down-regulated in the liver of diabetic control rats, when compared with normal control rats, but was significantly upregulated after treatment with farrerol. This may be due to dysfunctional hepatocyte nuclear factor $4 \alpha$ 
(HNF4a), and is in agreement with those of previous studies [14]. It is likely that GLUT2 was responsible for the normalized glycemic levels in farrerol-treated diabetic rats. It has been reported that Akt-mediated GLUT2 enhancement in liver is responsible for the amelioration of hyperglycemia in STZ-induced DM [15].

\section{CONCLUSION}

The results of this study show that farrerol alleviates STZ-induced hyperglycemia and dyslipidemia in rats via reduction in oxidative stress and inflammation, and upregulation of GLUT2 protein expression. Thus, farrerol has antidiabetic and hepatoprotective potentials for therapeutic applications in humans.

\section{DECLARATIONS}

\section{Conflict of interest}

No conflict of interest is associated with this work.

\section{Contribution of authors}

We declare that this work was done by the authors named in this article and all liabilities pertaining to claims relating to the content of this article will be borne by the authors. All authors equally contributed to the study design, animal studies and manuscript writing. All authors read and approved the final manuscript for publication.

\section{Open Access}

This is an Open Access article that uses a funding model which does not charge readers or their institutions for access and distributed under the terms of the Creative Commons Attribution License (http://creativecommons.org/licenses/by/ 4.0) and the Budapest Open Access Initiative (http://www.budapestopenaccessinitiative.org/rea d), which permit unrestricted use, distribution, and reproduction in any medium, provided the original work is properly credited.

\section{REFERENCES}

1. Kaiser AB, Zhang N, Pluijm WVD. Global Prevalence of Type 2 Diabetes over the Next Ten Years (2018-2028). Diabetes 2018; 67 (Supplement 1).

2. Mantovani A, Targher $G$. Type 2 diabetes mellitus and risk of hepatocellular carcinoma: spotlight on nonalcoholic fatty liver disease. Ann Trans/ Med 2017; 5(13): 270

3. Dharmalingam M, Yamasandhi PG. Nonalcoholic Fatty Liver Disease and Type 2 Diabetes Mellitus. Indian J Endocrinol Metab 2018; 22(3): 421-428.

4. Athyros VG, Polyzos SA, Kountouras J, Katsiki N, Anagnostis $P$, Doumas M, Mantzoros CS. Non-alcoholic fatty liver disease treatment in patients with type 2 diabetes mellitus; new kids on the block. Curr Vasc Pharmacol 2019; "In press".

5. Qin X, Hou X, Zhang K, Li Q. Farrerol Modulates Aorta Gene Expression Profile in Spontaneously Hypertensive Rats. Planta Med 2018; 84(5): 296-303.

6. Wang L, Wei W, Xiao Q, Yang H, Ci X. Farrerol Ameliorates APAP-induced Hepatotoxicity via Activation of Nrf2 and Autophagy. Int J Biol Sci 2019; 15(4): 788799.

7. Guide for the Care and Use of Laboratory Animals: 8th Edn. $\quad$ https://www.nap.edu/read/12910/chapter/1. Accessed 2 April 2019

8. Jiang F, Lim HK, Morris MJ, Prior L, Velkoska E, Wu X, Dusting GJ. Systemic upregulation of NADPH oxidase in diet-induced obesity in rats. Redox Rep 2011; 16(6): 223-229.

9. Tiwari BK, Pandey KB, Abidi AB, Rizvi SI. Markers of Oxidative Stress during Diabetes Mellitus. J Biomark 2013; 2013: 378790.

10. Zhang $Q$, Deng Q, Zhang J, Ke J, Zhu Y, Wen RW, Ye Z, Peng H, Su ZZ, Wang C, Lou T. Activation of the Nrf2ARE Pathway Ameliorates Hyperglycemia-Mediated Mitochondrial Dysfunction in Podocytes Partly Through Sirt1. Cell Physiol Biochem 2018; 48(1): 1-15.

11. Chadt A, Scherneck S, Joost HG, Al-Hasani H. Molecular links between obesity and diabetes: "Diabesity". de Groot LJ, Beck-Peccoz P, Chrousos G, Dungan K, Grossman A, Hershman JM, Koch C, McLachlan R, New M, Rebar R, et al., Eds.; MDText.com, Inc.: South Dartmouth, MA, USA, 2000.

12. Wen $H$, Gris $D$, Lei $Y$, Jha $S$, Zhang L, Huang $M T$, Brickey WJ, Ting JP. Fatty acid-induced NLRP3-ASC inflammasome activation interferes with insulin signaling. Nat Immunol 2011; 12(5): 408-415.

13. Han LP, Li CJ, Sun B, Xie Y, Guan Y, Ma ZJ, Chen LM. Protective Effects of Celastrol on Diabetic Liver Injury via TLR4/MyD88/NF-KB Signaling Pathway in Type 2 Diabetic Rats. J Diabetes Res 2016; 2016: 2641248.

14. Stoffel M, Duncan SA. The maturity-onset diabetes of the young (MODY1) transcription factor HNF4alpha regulates expression of genes required for glucose transport and metabolism. Proc Natl Acad Sci USA. 1997; 94(24): 13209-13214.

15. Rathinam A, Pari L. Myrtenal ameliorates hyperglycemia by enhancing GLUT2 through Akt in the skeletal muscle and liver of diabetic rats. Chem Biol Interact 2016; 256 : 161-166. 